Document No. Y/DK-2176 R1

A BWXT/Bechtel Enterprise

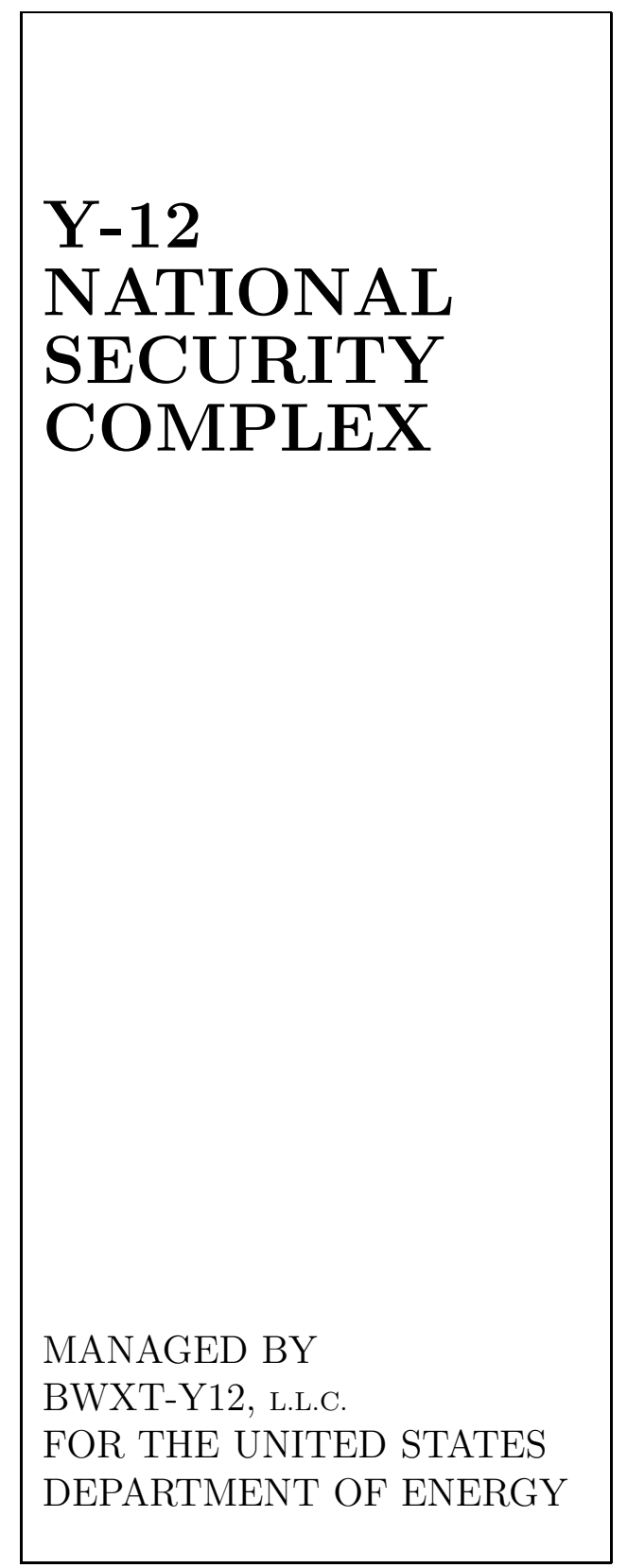

Far-Field Approximation in the Generalized Geometry Holdup (GGH) Model
R. B. Oberer
C. A. Gunn
L. G. Chiang

September 7, 2006

Prepared by the

Y-12 National Security Complex

P.O. Box 2009

Oak Ridge, TN 37831-8169

managed by

BWXT-Y12, L.L.C.

for the

U.S. DEPARTMENT OF ENERGY

under contract DE-AC05-00OR22800 


\section{DISCLAIMER}

This report was prepared as an account of work sponsored by an agency of the United States Government. Neither the United States Government nor any agency thereof, nor any of their employees, makes any warranty, express or implied, or assumes any legal liability or responsibility for the accuracy, completeness, or usefulness of any information, apparatus, product, or process disclosed, or represents that its use would not infringe privately owned rights. Reference herein to any specific commercial product, process, or service by trade name, trademark, manufacturer, or otherwise does not necessarily constitute or imply its endorsement, recommendation, or favoring by the United States Government or any agency thereof. The views and opinions of authors expressed herein do not necessarily state or reflect those of the United States Government or any agency thereof.

\section{COPYRIGHT NOTICE}

The submitted manuscript has been authored by a subcontractor of the U. S. Government under contract DE-AC05-00OR-22800. Accordingly, the U. S. Government retains a paid-up, nonexclusive, irrevocable, worldwide license to publish or reproduce the published form of this contribution, prepare derivative works, distribute copies to the public, and perform publicly and display publicly, or allow others to do so, for $\mathrm{U}$. S. Government purposes. 


\title{
Far-Field Approximation in the Generalized Geometry Holdup (GGH) Model
}

\author{
R. B. Oberer \\ C. A. Gunn \\ L. G. Chiang
}

September 7, 2006

\begin{abstract}
Quantitative gamma spectrometry measurements of uranium frequently require corrections for attenuation by an equipment or container layer and by the uranium bearing material itself. It is common to correct for attenuation using the "far-field approximation". Under this approximation, the minimum thickness of equipment or material is used for the correction rather than an average thickness over the detector field-of-view. In reality this aspect of the far-field approximation is really a narrow field-of-view approximation. The price of this simplification is the introduction of a bias. This bias will be investigated in this paper. In addition, there is a distance dependence of the radial response of a detector. This dependence will also be investigated.
\end{abstract}

\section{Contents}

1 Introduction

2 Narrow Field-of-view from the GGH Radial Response

3 Experimental Verification of the Radial Transmission

5 Conclusion 


\section{Introduction}

Quantitative gamma spectrometry measurements of uranium are typically used to determine either a quantity of material or alternatively to determine the enrichment of the uranium. The measurements frequently require corrections for attenuation by an equipment or container layer and by the uranium bearing material. In the cases of both holdup measurements using the generalized geometry holdup (GGH) model $^{1}$ and enrichment measurements, ${ }^{2}$ it is common to correct for attenuation using the "far-field approximation." ${ }^{3}$ Under this approximation, the minimum thickness of equipment or material, perpendicular to the detector line-of-sight $\left(x_{m}\right.$ and $x_{e}$ as shown in Figure 1) is used for the correction rather than an average thickness over the detector field-of-view.

This aspect of the far-field approximation is really a narrow field-of-view approximation. A collimator restricts the field-of-view of a detector as shown in Figure 1. Although the detector sees various thicknesses of both equipment and material depending on the radial position of the source, the assumption allows the attenuation to be characterized as a function of one thickness only. The price of this simplification is the introduction of a bias. The magnitude of the bias from the narrow field-of-view approximation will first be quantified and corrected using the radial response from GGH. Following the discussion on the narrow field-of-view, the distance dependence of the detector response will be investigated.

\section{Narrow Field-of-view from the GGH Radial Response}

The magnitude of the bias can be understood in terms of the GGH radial response of the detector. A radial response is measured as part of the GGH calibration in holdup measurements. The radial response of the detector depends on the geometry of the calibration standard used to produce it. For a spherical or cylindrical standard there is no position dependence of the attenuation. For a sealed parallel slab standard, there is a position dependence for both equipment and self attenuation. The parallel slab geometry is a common geometry for both holdup measurements and enrichment measurements. For a holdup measurement, if the equipment thickness and material thickness of both the standard and unknown are identical, the narrow field-of-view assumption is correct. This observation is the key to adjusting the attenuation correction for a wide field-of-view. The calibration standard

\footnotetext{
${ }^{1}$ The most current description of this method can be found in P. A. Russo, Gamma-Ray Measurements of Holdup Plant-Wide: Application Guide for Portable, Generalized Approach, Los Alamos Technical Report LA-14206, June 2005.

${ }^{2}$ Doug Reilly, Norbert Ensslin, Hastings Smith, Jr. and Sara Kreiner, Passive Nondestructive Assay of Nuclear Materials, NUREG/CR-5550, March 1991, pp. 195-219.

${ }^{3}$ Far-field approximations require that the detector is far enough away from the material being measured such that all the paths from the material to the detector are of equal length and parallel to each other.
} 


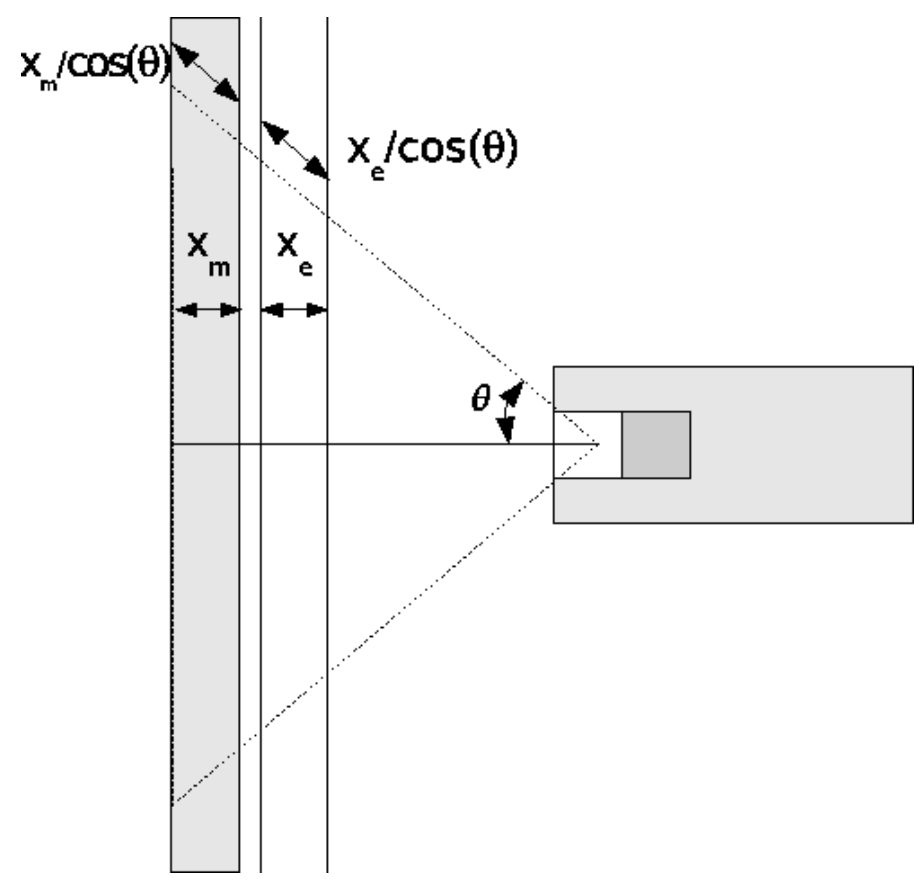

Figure 1: Geometry showing the violation of the narrow field-of-view assumption for line and area sources.

does not need to actually match the unknown. Instead the radial response of the identical calibration standard can be predicted theoretically from an observed radial response.

The first step in making the wide-field correction is to eliminate the position dependent attenuation from the radial response of the detector for the calibration standard used. Next the radial response for varying thicknesses of equipment and material are calculated. From these radial responses, varying effective areas $A$ and lengths $L$ are then calculated.

The first two steps are performed by converting each point in the normalized radial response from the calibration standard $R_{i s}$ to an equivalent calibration standard $R_{i}\left(T_{m}, T_{e}\right)$ of material transmission $T_{m}$ and equipment transmission $T_{e}$.

$$
R_{i}\left(T_{m}, T_{e}\right)=R_{i s} \frac{\ln \left(T_{m s i}\right)}{\left(1-T_{m s i}\right) T_{e s i}} \frac{\left(1-T_{m i}\right) T_{e i}}{\ln \left(T_{m i}\right)}
$$

The measured radial response of the detector set and standard $R_{i s}$ is performed once at calibration. The factor $\frac{\ln \left(T_{m s i}\right)}{\left(1-T_{m s i}\right) T_{e s i}}$ for each point in the radial response is a property of the standard. The factor $\frac{\left(1-T_{m i}\right) T_{e i}}{\ln \left(T_{m i}\right)}$ depends on the transmission of the unknown material and equipment. The ith transmission $T_{i}$ is related to the zeroth transmission $T_{0}$ by $T_{i}=$ $e^{\ln \left(T_{0}\right) / \cos \left(\theta_{i}\right)}$. Unfortunately, the final quantities $L\left(T_{m}, T_{e}\right)$ and $A\left(T_{m}, T_{e}\right)$, from GGH, are weighted sums of each of the $i$ radial responses, e.g., $A\left(T_{m}, T_{e}\right)=\sum_{i=0}^{N} a_{i} R_{i}\left(T_{m}, T_{e}\right)$. The effective length, $L\left(T_{m}, T_{e}\right)$, is calculated in an analogous manner. 


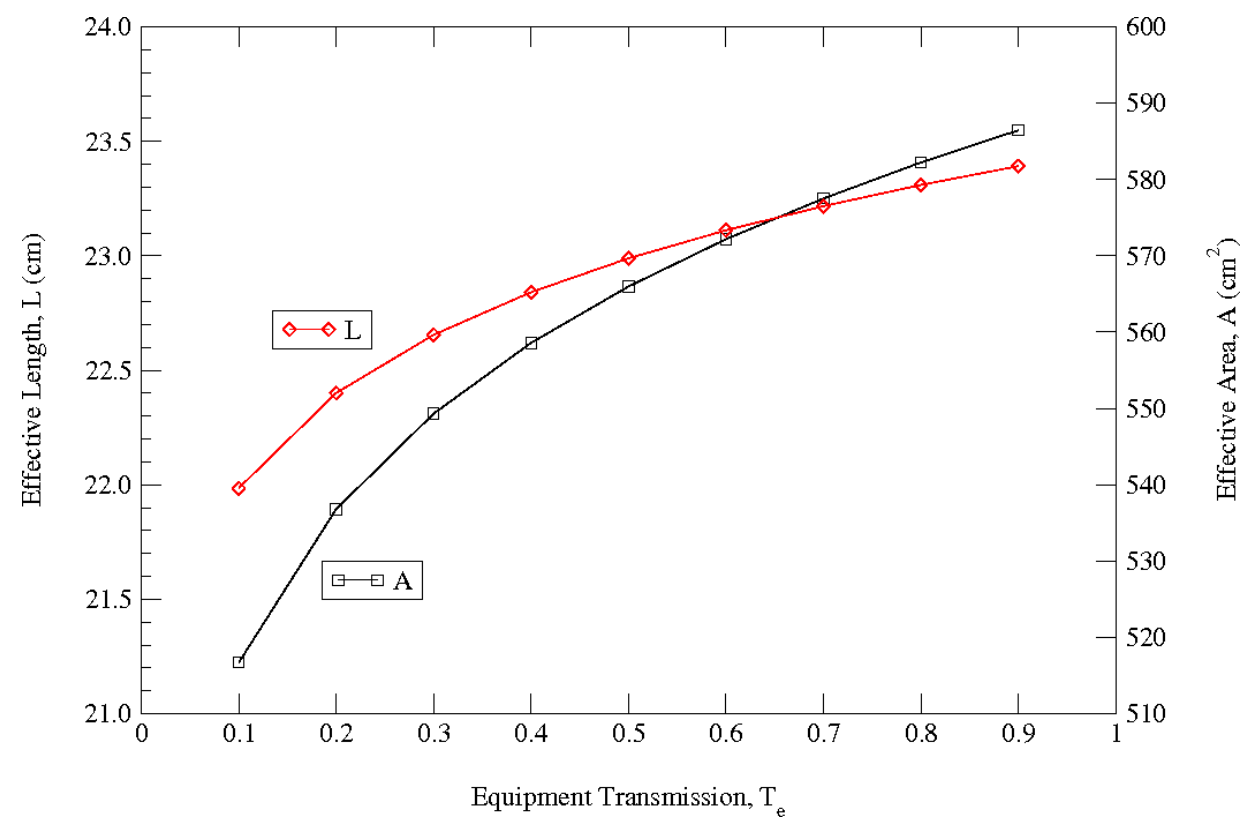

Figure 2: The effective area and length as a function of equipment transmission with a material transmission of 1 .

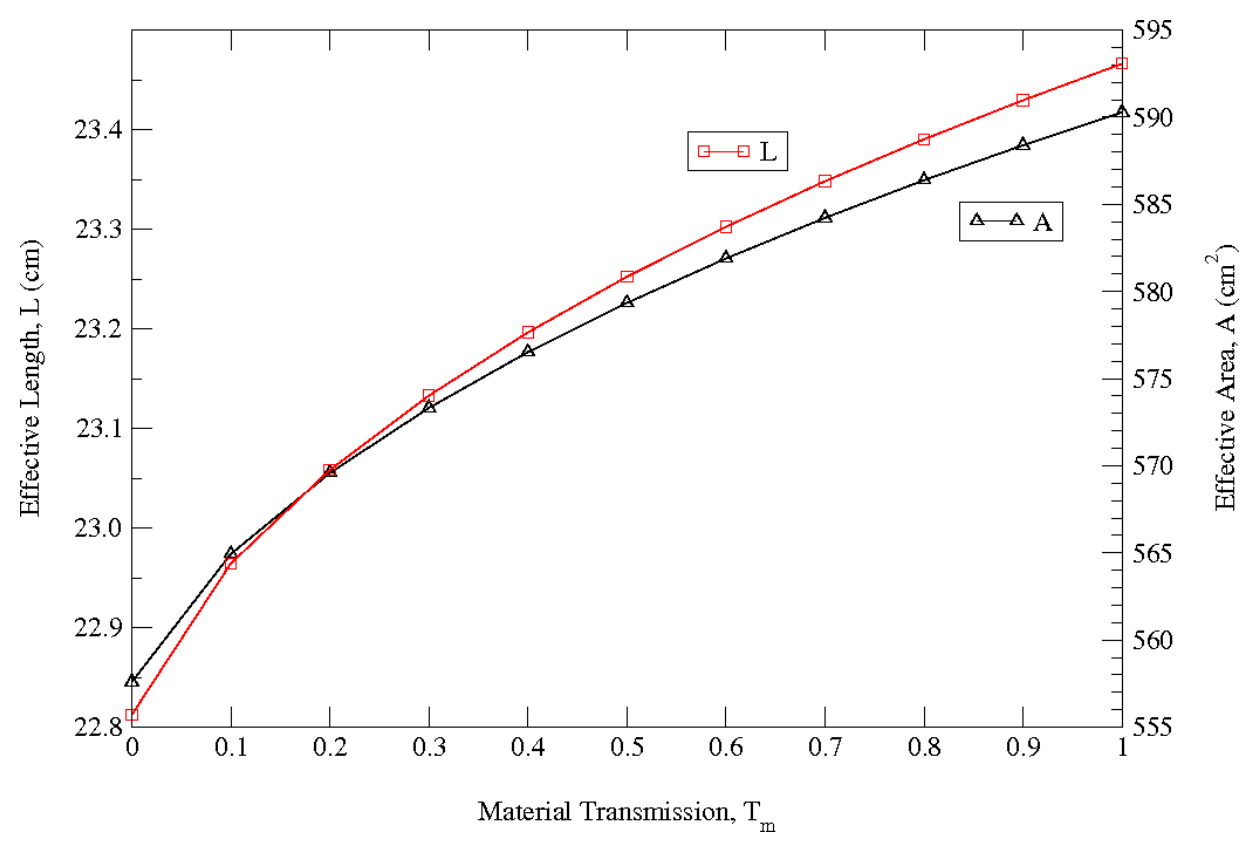

Figure 3: The effective area and length as a function of material transmission with no equipment layer, i.e. equipment transmission equals 1 . 
The effective length $L\left(T_{m}, T_{e}\right)$ and effective area $A\left(T_{m}, T_{e}\right)$ for a typical detector are plotted in Figures 2 and 3. In Figure 2 the material transmission is set to 1 and the equipment transmission varied from 0.1 to 1 . In Figure 3 the material transmission varies with no equipment layer (equipment transmission set to 1). Figure 3 is plotted again in Figure 4, normalized to the full transmission effective length, $L(1,1)$ and effective area, $A(1,1)$. From Figure 4 the total magnitude of the narrow field-of-view assumption is roughly $5 \%$. If the equipment transmission is also reduced to $10 \%$, the effective area goes down to $83 \%$ and the effective length goes down to $91 \%$. This extreme case probably establishes the boundary on the magnitude of the potential bias of the narrow field-of-view assumption. Whether these errors represent random or systematic errors depends on the geometry of the standard used for the radial response and the transmission of the material and cladding of the standard.

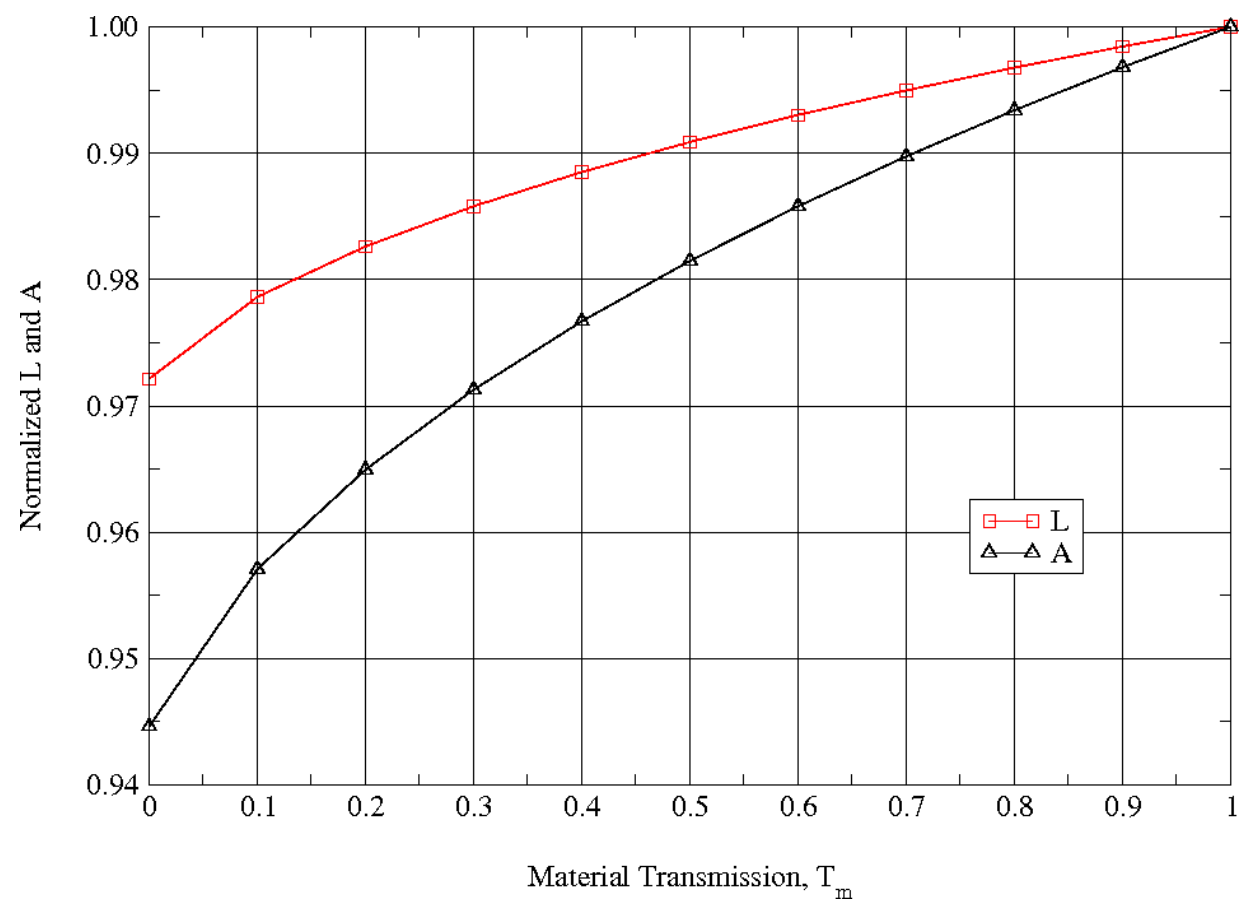

Figure 4: The proportion of full transmission effective area and length as a function of material transmission with no equipment layer.

The use of the effective area, adjusted for the wide field-of-view, can be used by recalling 
that the detector response is

$$
\begin{aligned}
R & =\frac{\epsilon f_{235} A I}{\mu_{m}}\left(1-e^{-\mu_{m} \rho_{m} x_{m}}\right) e^{-\mu_{e} \rho_{e} x_{e}} \\
& =\frac{\epsilon f_{235} A I}{\mu_{m}}\left(1-T_{m}\right) T_{e} \\
& =\frac{\epsilon f_{235} A I \rho_{m} x_{m}}{\ln \left(T_{m}\right)}\left(1-T_{m}\right) T_{e} \\
& =\frac{f_{235} \rho_{m} x_{m}}{K_{a}} \frac{\left(1-T_{m}\right) T_{e}}{\ln \left(T_{m}\right)}
\end{aligned}
$$

where $f_{235} U$ is the fraction of ${ }^{235} U$ to material, $I$ is the specific emission rate, $\epsilon$ is the detection efficiency, $A$ is the effective area as discussed above, $\mu$ is the mass attenuation coefficient, $\rho$ is the density, and $x$ is the thickness of the material $m$ and equipment $e$ respectively. With Equation (1), the narrow field-of-view assumption can be restated. Without the narrow field-of-view assumption, $A$ is a function of the transmission variables $\mu, \rho$, and $x$. The assumption allows us to treat $A$ as independent of $\mu, \rho$, and $x$.

Suppose a detector is calibrated to measure the mass ratio of ${ }^{235} U$ to total $U$ with a standard with a transmission $T_{m} s$ and cladding with a transmission $T_{s e}$. Now material is measured which has a transmission $T_{m}$ and cladding with a transmission $T_{e}$. Using the narrow fieldof-view assumption, the measured enrichment is adjusted by a correction factor

$$
C F=\frac{\mu_{m} f_{U s}\left(1-T_{m s}\right) T_{e s}}{\mu_{m s} f_{U m}\left(1-T_{m}\right) T_{e}}
$$

where $f_{U s}$ and $f_{U m}$ is the grams of uranium to material for the standard $s$ and unknown material $m$ respectively.

Typically the transmissions in the correction factor assumes a narrow field-of-view and are calculated using the relation $T=e^{-\mu \rho x}$. When this is done, the transmissions are slightly over estimated. A wide field-of-view (wfv) correction can be applied to remove this bias. This correction factor is

$$
C F_{\mathrm{wfv}}=\frac{A\left(T_{m}, T_{e}\right)}{A\left(T_{m s}, T_{e s}\right)}
$$

Enrichment measurements are typically made at contact. The proposed correction for a wide field-of-view assumes a point detector model. In other words, there is a single direction between a point source and the detector. When the point source is close to the detector, a single direction no longer describes the geometry. Instead there is a distribution of directions from the source to the detector. This issue is addressed in the section on the distance dependence of the radial response. 


\section{Experimental Verification of the Radial Transmis- sion}

An experiment was performed to verify that the transmission behaved according to $T_{i}=$ $e^{\ln \left(T_{0}\right) / \cos \left(\theta_{i}\right)}$ as a function of angle. An 11g holdup standard ${ }^{4}$, Std. 006, was placed $40 \mathrm{~cm}$ from the detector perpendicular to the axis of the detector in the normal $R_{0}$ position. The count rate of the detector was then recorded as a function of the angle of rotation of the standard from the normal orientation. This data is shown in Figure 5. The experiment was repeated twice. The detector response was the difference of two regions of interest. The second set of data was also analyzed with NaIGEM to determine the detector response instead of the two ROI method. ${ }^{5}$ This data is also plotted. This experimental data is compared with the theoretical

$$
R_{i}=R_{o} \frac{\ln \left(T_{m 0}\right)}{\left(1-T_{m 0}\right) T_{e 0}} \frac{\left(1-T_{m i}\right) T_{e i}}{\ln \left(T_{m i}\right)}
$$

For the $11 \mathrm{~g}$ standard, $T_{m 0}=0.1537$ and $T_{e 0}=0.8302$ and of course $T_{i}=e^{\ln \left(T_{0}\right) / \cos \left(\theta_{i}\right)}$. The discrepancy between the theoretical and measured is somewhat troubling. The general shape of the experimental data seems to verify the theoretical values. The discrepancy seems to be attributable to an anomaly in the standard rather than a deficiency in the predicted data. Incorrect values in either $T_{m 0}$ or $T_{e 0}$. could account of the difference. For example the values 0.20, 0.95 make the predicted curve match the data. The orientation of the actual uranium source in the stainless steel encapsulation might also cause the discrepancy.

\section{Detector Distance Dependence of the Radial Re- sponse}

In addition to the narrow field-of-view, there is a source-to-detector distance dependence on the radial response of the detector. Figure 6 shows the radial response of a detector from contact with the collimator to $40 \mathrm{~cm}$ from the detector face. The radial response was simulated with MCNPX. ${ }^{6}$ The detector was modeled as a 1 inch by half inch thick cylindrical $\mathrm{NaI}$ detector. The collimator was a lead cylinder with a 1 inch by 1 inch aperture. Although the radial response changes dramatically with distance, the calibration constant $K_{a}$ remains relatively independent of distance as shown in Figure 7. At close distances, there is a

\footnotetext{
${ }^{4}$ The nominal $11 \mathrm{~g}$ standard consists of a $4.762 \mathrm{~cm}$ diameter, circular deposit of $10.572 \mathrm{~g}$ of ${ }^{235} \mathrm{U}$ encased in stainless steel, in a parallel slab geometry.

${ }^{5} \mathrm{NaIGEM}$ uses various curve-fitting functions to resolve the spectrum.

${ }^{6}$ D. B. Pelowitz, ed., MCNPX Users Manual, Version 2.5.0, Los Alamos National Laboratory, Report LA-CP-05-0369, April 2005
} 


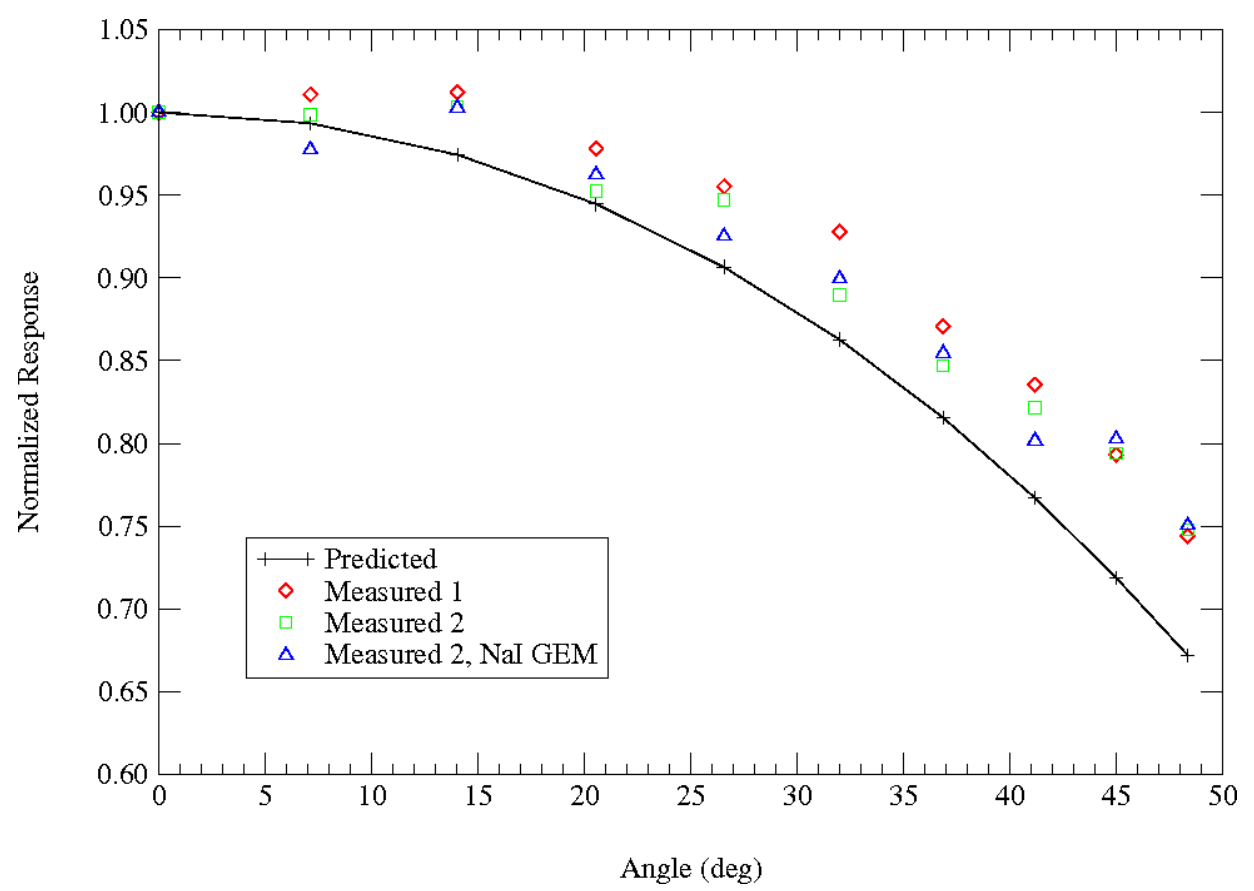

Figure 5: The predicted versus the measured affect of the angle in the wide field-of-view.

distribution of directions from the point source to the detector. Therefore the transmission as a function of position no longer behaves as $T_{i}=e^{\ln \left(T_{0}\right) / \cos \left(\theta_{i}\right)}$. Another problem at close distances is that it becomes difficult to reduce equipment transmission without changing the source to detector distance. For an on contact measurement it is not possible to interpose an equipment layer without increasing source to detector distance. At these close distances the simple wide field-of-view correction from Equation (2) should probably be avoided.

Because of the troubling distance dependence of the radial response, two additional tests were made of the distance independence of the detector response from an area source. The first was a measurement of an alpha standard (SS35U0135, 174,581 dpm). A small quantity of 93\% enriched uranium is plated within a 1.5 inch diameter circle on a 2 inch diameter planchet. Presumably the uranium is uniformly deposited within the 1.5 inch circle. The advantage of this source is that there is negligible equipment of self attenuation. A disadvantage is that the count rate is very low. Even though the measurement of this standard was performed in the Old Lung Counter to minimize contributions from background, the count rate was only slightly above background. Because of these disadvantages and no guarantee of uniformity, the distance independence of the count rate is not conclusive. Results are shown in Figure 8.

A similar experiment was performed with a 255 gram, infinitely thick, $93 \%$ enriched, enrichment standard (U235-0531). This standard is approximately 3 inches in diameter. The results of this experiment are shown in Figure 9. The count rate variation in this measurement is less than $1 \%$. In addition there is no evidence of distance dependence. 


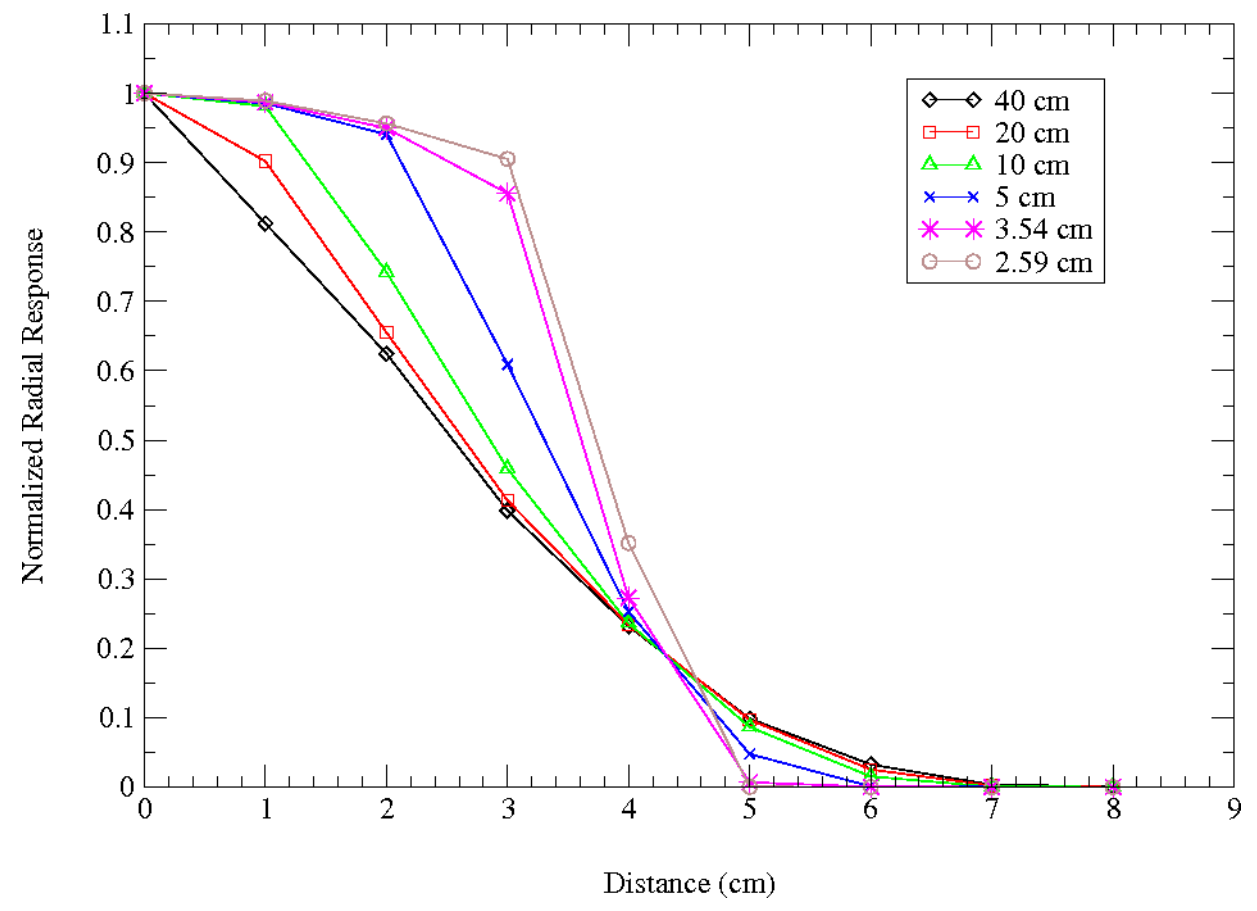

Figure 6: The radial response as a function of distance for a half inch detector with a 1 by 1 inch cylindrical collimator. The minimum distance of $2.59 \mathrm{~cm}$ is in contact with the collimator.

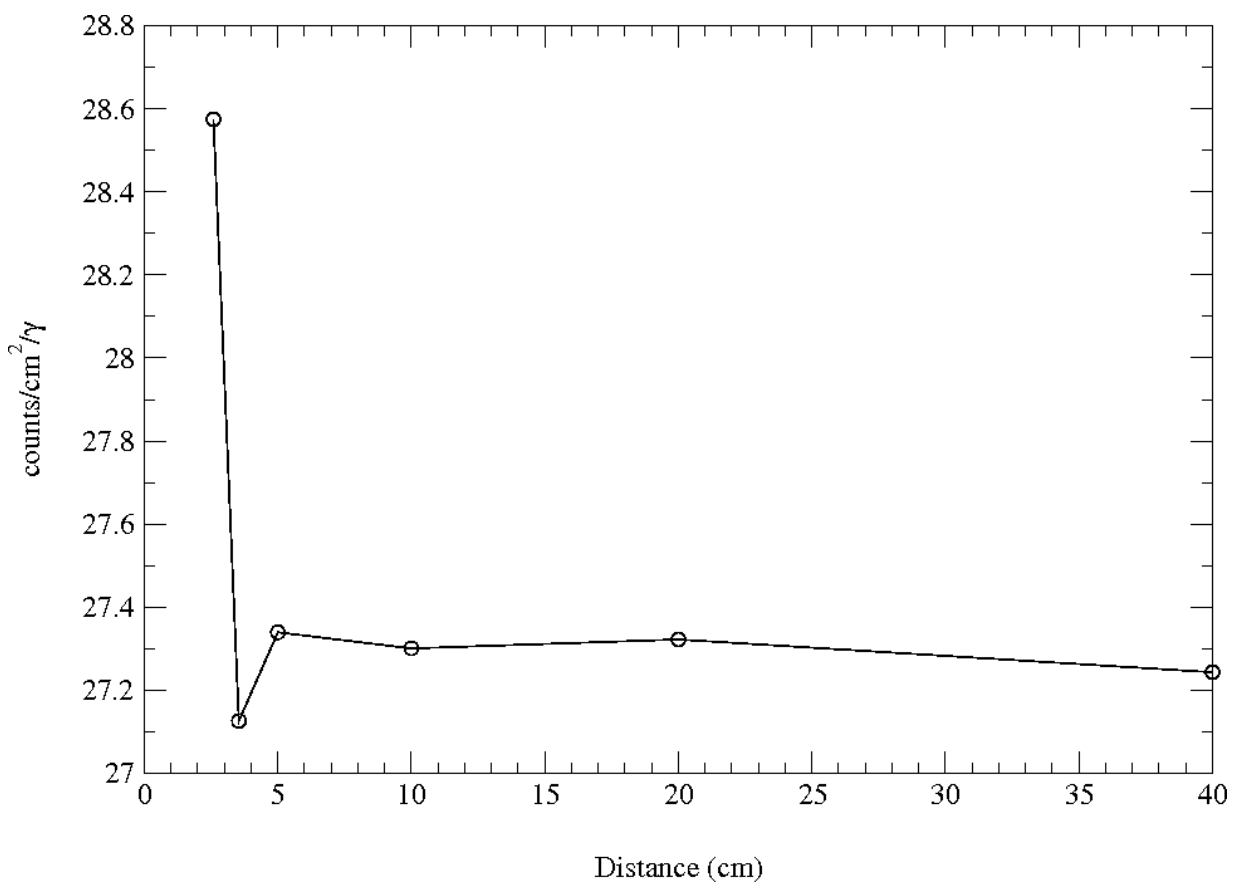

Figure 7: Calibration constant $K_{a}$ per $186 \mathrm{keV}$ gamma ray as a function of distance measured from the detector. 


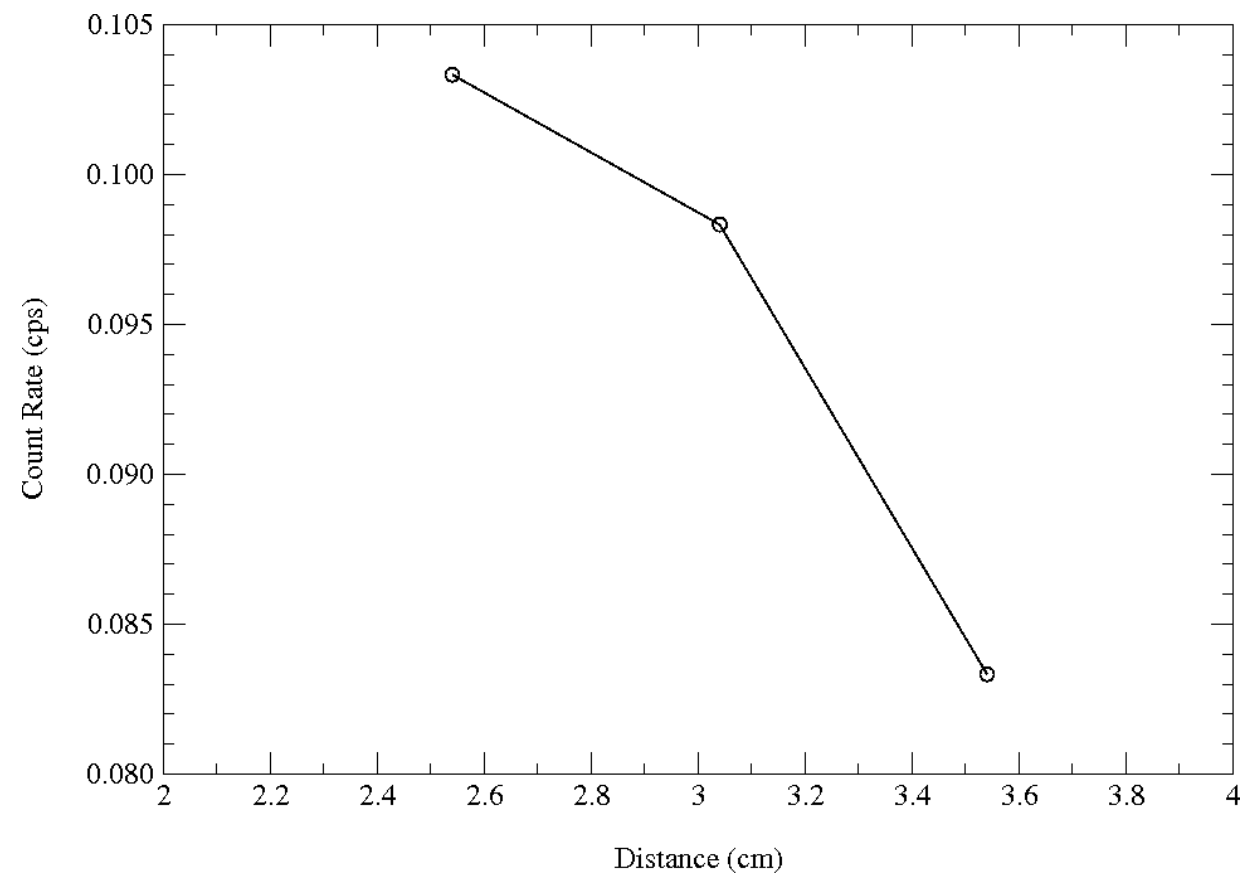

Figure 8: Alpha standard SS35U0135, 174,581 dpm standard plated on a 1.5 inch diameter circle on a 2 inch planchet.

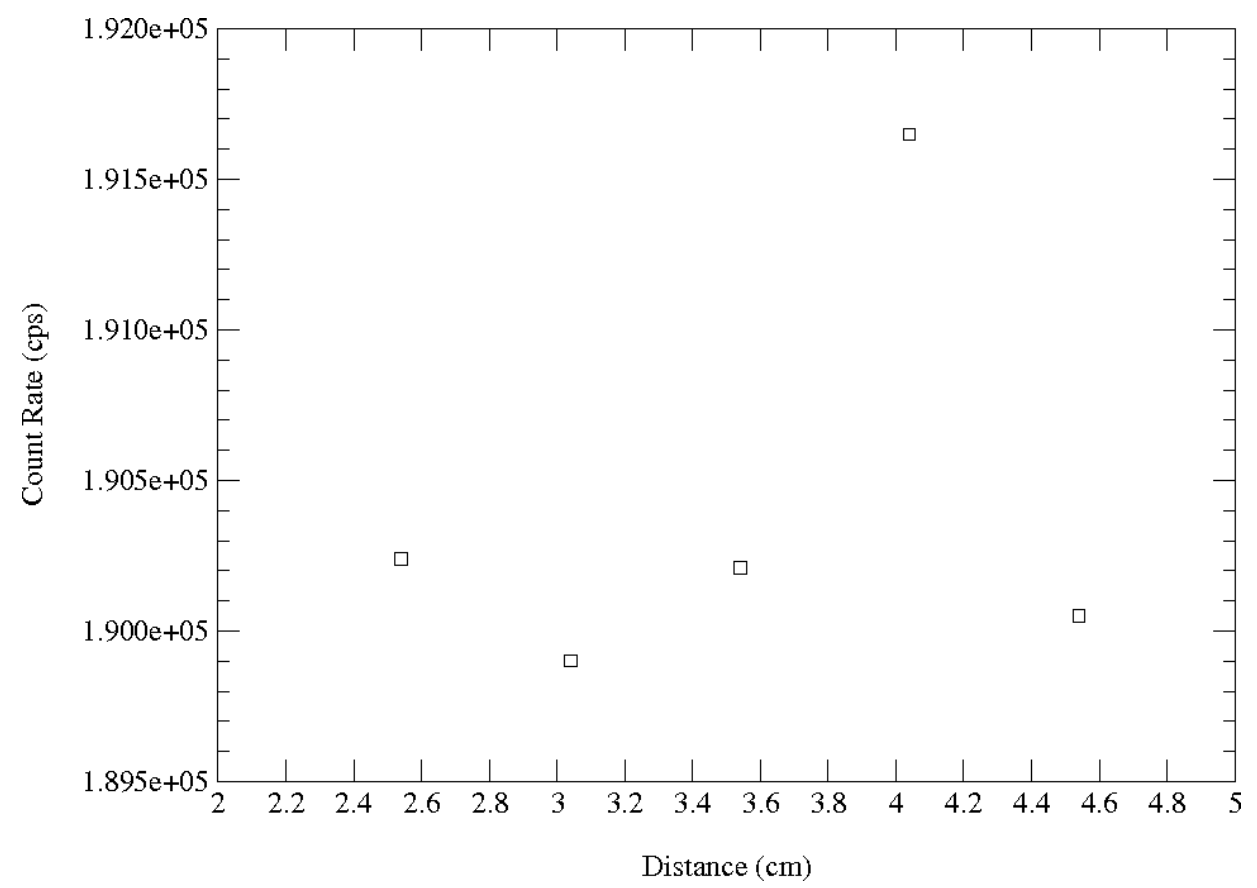

Figure 9: Y-12 NMC\&A enrichment standard U235-0531, 93.16\% ${ }^{235} \mathrm{U}, 255 \mathrm{~g}$ "infinitely thick" metal standard. 


\section{Conclusion}

The far-field assumption is really a combination of a narrow field-of-view assumption as well as a distance assumption. The far-field assumption breaks down at distances less than $10 \mathrm{~cm}$. Nevertheless the area calibration seems to hold. The point and line calibrations should not hold up as well. At distances of greater than $10 \mathrm{~cm}$, the narrow field-of-view assumption causes a small error. Whether this error is a bias or random depends on the calibration standard used to measure the radial response of the detector. The maximum bias results from thin, cylindrical or spherical standards. A parallel slab standard with average

equipment and material thickness provides the least bias. The maximum error is on the order of 10 to $15 \%$. 